\title{
Decision analytic model for evaluation of suspected coronary disease with stress testing and coronary CT angiography.
}

\author{
Ethan J Halpern \\ Thomas Jefferson Univerity \\ David Fischman \\ Thomas Jefferson Univerity \\ Michael P Savage \\ Thomas Jefferson Univerity \\ Anish R Koka \\ Thomas Jefferson Univerity \\ Moptthew \\ Thomas Jefferson Univerity \\ Part of the Radiology Commons

\section{Let us know how access to this document benefits you}

\section{Recommended Citation}

Halpern, Ethan J; Fischman, David; Savage, Michael P; Koka, Anish R; DeCaro, Matthew; and Levin, David C, "Decision analytic model for evaluation of suspected coronary disease with stress testing and coronary CT angiography." (2010). Department of Radiology Faculty Papers. Paper 9.

https://jdc.jefferson.edu/radiologyfp/9

This Article is brought to you for free and open access by the Jefferson Digital Commons. The Jefferson Digital Commons is a service of Thomas Jefferson University's Center for Teaching and Learning (CTL). The Commons is a showcase for Jefferson books and journals, peer-reviewed scholarly publications, unique historical collections from the University archives, and teaching tools. The Jefferson Digital Commons allows researchers and interested readers anywhere in the world to learn about and keep up to date with Jefferson scholarship. This article has been accepted for inclusion in Department of Radiology Faculty Papers by an authorized administrator of the Jefferson Digital Commons. For more information, please contact: JeffersonDigitalCommons@jefferson.edu. 


\section{Authors}

Ethan J Halpern, David Fischman, Michael P Savage, Anish R Koka, Matthew DeCaro, and David C Levin 
As submitted to:

Academic Radiology

And later published as:

"Decision Analytic Model for Evaluation of Suspected Coronary Disease with Stress Testing and Coronary CT Angiography"

\author{
Volume 17, Issue 5, May 2010, Pages 577-586 \\ DOI: 10.1016/j.acra.2009.12.015
}




\begin{abstract}
Rationale and Objectives: To apply a decision analysis model for evaluation of coronary artery disease (CAD) to define optimal utilization of cCTA and stress testing.
\end{abstract}

Materials and Methods: Our model assumes CAD is evaluated with a stress test and/or cCTA, and that a patient with a positive evaluation undergoes cardiac catheterization. Based upon values of sensitivity, specificity and radiation dose from the published literature and test costs from the Medicare fee schedule, a decision tree model was constructed as a function of disease prevalence.

Results: False negative rate (FNR) is lowest when cCTA is used as an isolated test. False positive rate (FPR) is minimized when cCTA is employed in combination with stress echocardiography. Effective radiation is minimized by use of stress ECG or stress echocardiography alone, or prior to cCTA. When the pretest probability of CAD is low, a strategy that employs stress echocardiography followed by cCTA minimizes FPR and effective radiation exposure, with relatively low imaging costs and with a FNR only slightly higher than a stress myocardial scintigraphy strategy. As the pretest probability of CAD increases above $20 \%$, the FNR of stress echocardiography followed by cCTA increases by more than 5\% relative to cCTA alone.

Conclusion: Effective radiation dose and imaging costs for the work-up of CAD may be minimized by an appropriate combination of stress testing and cCTA. A strategy that employs stress echocardiography followed by cCTA is appropriate for evaluation of the low risk CAD patient with a pre-test probability less than $20 \%$, while cCTA alone may be more appropriate in the intermediate risk patient. 
Key words: CT angiography, coronary artery disease, radiation exposure, imaging cost, costeffectiveness 


\section{Introduction}

Although the age-adjusted death rate from heart disease has been declining slowly over the past 50 years, heart disease remains the most common cause of death in the United States, and was responsible for $26 \%$ of all deaths in $2006 .{ }^{1}$ Atherosclerotic disease of the coronary arteries remains the most important etiology of heart disease. The evaluation of suspected coronary artery disease (CAD) should always begin with an appropriate history, physical examination and ECG. In the stable, symptomatic patient evaluation for CAD often progresses to non-invasive stress testing. The stress test provides a physiological evaluation of cardiac function and serves as a diagnostic study and prognostic marker for future coronary events. ${ }^{2}$

Coronary computed tomographic angiography (cCTA) provides a noninvasive alternative to cardiac catheterization for visualization of coronary anatomy, and has demonstrated high sensitivity and specificity for the anatomic presence of CAD. ${ }^{3,4}$ Although functional information about wall motion can be obtained during cCTA, the primary utility of cCTA is for imaging of the coronary arteries to define the presence of CAD. Stress testing provides additional information to predict the functional relevance of coronary stenosis with respect to myocardial ischemia. ${ }^{5,6}$ When cCTA demonstrates coronary stenosis of uncertain hemodynamic significance, a follow-up stress test may evaluate the functional significance of CAD found by cCTA. Given the divergent emphasis of stress testing on functional physiology and cCTA on coronary anatomy, these two modalities may provide complementary information in the evaluation of $\mathrm{CAD} .^{7}$

Stress testing has served as the traditional test of choice to evaluate symptoms that may be related to CAD. Three broad categories of stress testing include stress ECG without imaging, stress echocardiography and the nuclear stress 
test - myocardial perfusion scintigraphy (stress MPS). Although cCTA may be more accurate than stress testing for evaluation of "hemodynamically significant" coronary stenosis ${ }^{8}$ and appropriateness criteria have been evaluated for the application of cCTA, ${ }^{9}$ the precise role of cCTA (versus stress testing or in combination with stress testing) in the work-up of CAD remains poorly defined.

In this study, we apply a theoretical decision analytic model to evaluate the diagnostic accuracy, effective radiation dose and imaging costs associated with evaluation of CAD using various combinations of cCTA and stress testing. Our goal is to define the strengths and weaknesses of various testing strategies that may be employed in order to optimize evaluation of the symptomatic patient with suspected obstructive CAD. 


\section{Methods:}

A decision tree was constructed to compare false negative rates, false positive rates, effective radiation exposure and imaging costs during the work-up of suspected coronary disease with stress testing and/or cCTA (figure 1). The decision tree begins with a decision node - the rectangular box - where a CAD work-up strategy is chosen. The work-up options include evaluation with a single stress test or by cCTA alone, as well as options for a combined evaluation with both stress testing and cCTA. Each individual test option is represented by a node - a circle whose outcome is governed by the sensitivity and specificity of that particular test. The work-up strategy follows the upper branch out of the test node when the test is positive, and the lower branch out of the test node when the test is negative. For combined evaluations, the decision tree includes options that begin with a stress test and proceed to cCTA as well as options that begin with cCTA and proceed to a stress test. In order to minimize unnecessary cardiac catheterizations, patients in this model are referred to catheterization only when non-invasive diagnostic testing is positive. When an option for combined testing is chosen, the patient is referred for the second test only if the first test is positive, and is referred to catheterization only when both the stress test and the cCTA are positive.

Spreadsheet computations for expected rates (or proportions) of true and false diagnoses among the population presenting for diagnostic testing were implemented as follows:

True positive rate $(\mathrm{TPR})=$ prevalence $\mathrm{x}$ sensitivity

False negative rate $(\mathrm{FNR})=$ prevalence - True positive rate

True negative rate $(\mathrm{TNR})=(1-$ prevalence $) *$ specificity

False positive rate $($ FPR $)=(1-$ prevalence $)-$ True negative rate 
It is important to distinguish the positive and negative rates/proportions in a study population (as defined above) from the positive and negative rates of a diagnostic examination. With respect to a diagnostic examination, the TPR (sensitivity), FNR (1-sensitivity), TNR (specificity) and FPR (1-specificity) are defined independent of disease prevalence. However, the expected proportion of positive and negative tests in a population and are dependent upon disease prevalence. For the purpose of health care policy decisions, the proportion of correct diagnoses in a study population (TPR + TNR), the proportion of incorrect diagnoses in a study population (FPR + FNR) and the overall imaging costs in the population are of interest. The TPR, TNR, FPR, and FNR of individual diagnostic tests are only important in so far as they impact the proportion of correct and incorrect diagnoses in the population. For the remainder of this manuscript all references to positive and negative rates refer to proportions within the study population.

Although CAD may be defined by gradations of severity, we use a dichotomous definition for CAD to simplify the decision analysis. cCTA is defined as positive for the presence of atherosclerotic disease when there is more than $50 \%$ diameter narrowing. The results of cardiac catheterization (our "gold standard") are assumed to be correct. Expected rates of true and false diagnoses are propagated through the decision tree based upon a Bayesian analysis that depends only upon prevalence of disease in the study population (= pre-test probability of disease for the individual patient) and the sensitivity/specificity of each test. At each testdecision node within the tree, the expected rate of positive exams is calculated as the sum of the true positive rate + false positive rate while the expected rate of negative exams is calculated as the sum of the true negative rate + false negative rate. 
For any particular work-up strategy in the decision tree, effective radiation dose is computed as the sum of the effective radiation dose for each test in the pathway multiplied by the proportion of patients who will be subjected to that test. Imaging cost is similarly computed as the sum of the imaging cost for each test in the pathway multiplied by the proportion of patients who will be subjected to that test. Since the cost of cardiac catheterization is far greater than the cost of stress testing and cCTA, a strategy that employs more testing can result in reduced imaging costs if the rate of cardiac catheterizations is reduced.

A range of values for the sensitivity and specificity of stress tests and cCTA are reported in the literature. The values used for this analysis are summarized in table 1 . The values for stress testing are based upon published meta analyses of the literature for stress ECG testing ${ }^{10}$ as well as stress echocardiography and stress MPS. ${ }^{11}$ The sensitivity and specificity of cCTA for CAD with diameter stenosis greater than $50 \%$ were based upon a recent multicenter trial by Budoff. ${ }^{12} \mathrm{~A}$ sensitivity analysis was performed by varying the sensitivity and specificity of cCTA according to values obtained in two other recent multicenter trials - one trial by Meijboom demonstrating lower specificity, ${ }^{13}$ and a second trial by Miller demonstrating lower sensitivity. ${ }^{14}$ Our primary analysis was based upon sensitivity/specificity data from the Budoff trial because these values are intermediate between those of Meijboom and Miller. Finally, although single center trials may overestimate the accuracy of a diagnostic study, several recent meta analyses of single center studies have suggested higher diagnostic accuracy than the multicenter trials referenced above., ${ }^{3,4}$ Therefore, higher values of sensitivity and specificity were also tested based upon the analysis of Vanhoenacker. ${ }^{3}$ 
Effective radiation dose for stress MPS, cCTA and diagnostic cardiac catheterization is a moving target with improvements in technology. Effective doses used for the current analysis are summarized in table 2. For stress MPS, the base value used for the analysis was $12 \mathrm{mSv}$, based upon the reported dose for a Tc$99 \mathrm{~m}$ sestamibi 1 -day rest-stress protocol. ${ }^{15}$ For the purpose of sensitivity analysis, the dose of a stress MPS examination was also varied down to $9.4 \mathrm{mSv}$ as reported in a more recent review of nuclear medicine studies. ${ }^{16}$ In the interest of using an effective dose that is obtained with the latest generally available technology, we assume a $4.2 \mathrm{mSv}$ effective dose for prospectively ECG-gated cCTA based upon clinical reports. ${ }^{17,18}$ For the purpose of sensitivity analysis, since many centers have not yet adopted prospectively gated cCTA, the effective dose of cCTA was also varied up to $10 \mathrm{mSv}$ as demonstrated in a recent multicenter trial using the more conventional helical CTA with tube current modulation. ${ }^{19}$ The effective dose for diagnostic cardiac catheterization was set at $7 \mathrm{mSv}$ for the base analysis, but was varied down to $5 \mathrm{mSv}$ for the sensitivity analysis. ${ }^{20,21}$

Imaging/testing costs were computed as a combination of professional and technical fees as reported in table 3. For the purpose of cost comparisons, we refer to these costs as imaging costs even though the stress ECG study does not include an imaging component. Professional and technical fees for stress testing, cCTA and diagnostic cardiac catheterization were obtained from the 2009 physician Medicare fee schedule provided by our local carrier (Highmark Medicare; Camp Hill, PA). These fees are very similar to the 2009 national average Medicare payments (based upon the Nov 2008 Federal Register using a 2009 conversion factor http://www.bostonscientific.com/Reimbursement). The facility fee for cardiac catheterization was not available in the 2009 physician medicare fee schedule 
provided by our local carrier, and was therefore based upon the national average facility fee from the Federal Register.

The decision tree model was implemented on a spreadsheet (Quattro Pro; Correl Corp. Ottawa, Ontario). Computed values of false negative rates, false positive rates, costs and radiation exposure were obtained for a disease prevalence (= pre-test probability) that ranged from 0.01 to 0.60 . The calculated values represent expected mean values as a function of CAD prevalence. Results of this analysis are presented in graphical format. 
Results:

The imaging cost and expected effective radiation dose associated with each of the various work-up strategies for CAD are depicted to the right side of each terminal branch of the decision tree in figure 1. Figures 2 and 3 demonstrate the false negative and false positive rates for the diagnosis of CAD as a function of CAD prevalence, using our baseline assumptions of sensitivity and specificity (Budoff). Table 4 reports the values of these positive and negative work-up rates as a function of the prevalence of CAD. The order in which the stress test and cCTA are performed make no difference in this calculation. False negative rate (FNR) is proportional to CAD prevalence while false positive rate (FPR) is inversely related to CAD prevalence. FNR is lowest when cCTA is used as an isolated test. When a stress test is used in combination with cCTA, the FNR is slightly higher compared with the FNR when the same stress test is used alone. Specifically, the FNR of a combined approach including cCTA is increased by up to $3 \%$ relative to the FNR of a stress test alone as the prevalence of CAD approaches $60 \%$. However, the FPR of the combined approach is reduced by $9-19 \%$ as the prevalence of CAD approaches 1\%. FPR is minimized when cCTA is employed in combination with stress echocardiography.

Figure 4 demonstrates expected effective radiation dose as a function of CAD prevalence. Strategies that employ no advanced imaging $(0.0 \mathrm{mSv})$ or proceed directly to cardiac catheterization $(7.0 \mathrm{mSv})$ are not demonstrated in this figure since the effective radiation dose for these strategies is independent of CAD prevalence. Strategies that use stress MPS result in a higher effective dose than all other strategies. Stress MPS alone results in an effective dose that is approximately $8 \mathrm{mSv}$ 
higher than cCTA alone. When a stress ECG or stress echocardiogram is added following cCTA, effective radiation dose is reduced by approximately $1 \mathrm{mSv}$ relative to cCTA alone. However, when a stress ECG or stress echocardiogram is added prior to cCTA, effective radiation dose is reduced by $4-5 \mathrm{mSv}$ because fewer cCTA studies are performed. A work-up strategy that employs stress ECG or stress echocardiography prior to cCTA minimizes effective radiation exposure compared to all other strategies that use cCTA and/or MPS. When the prevalence of CAD is below $47 \%$, a strategy that employs stress echocardiography followed by cCTA will result in an expected effective radiation dose that is below that of a single cCTA $(4.2 \mathrm{mSv})$. When the prevalence of CAD is below $10 \%$, a strategy that employs stress echocardiography followed by cCTA may actually result in an effective radiation dose that is lower than a strategy that uses stress echocardiography alone.

Overall imaging costs are summarized in figure 5 as a function of CAD prevalence. A strategy that tests all patients with cardiac catheterization is not demonstrated in figure 5 as this strategy would result in a fixed imaging cost of \$2948, independent of CAD prevalence. As long as the pre-test probability of CAD is below $75 \%$, the cost for each of the remaining strategies is below the cost of proceeding directly to cardiac catheterization. When the prevalence of CAD is below 45\%, a strategy that employs stress MPS together with cCTA will result in lower rates of cardiac catheterization and lower imaging costs as compared to a strategy that uses either MPS or cCTA alone. Imaging costs are minimized by a strategy that employs stress ECG followed by cCTA. This combined strategy saves at least $\$ 400$ per patient relative to a strategy that combines stress MPS with cCTA. From the imaging cost perspective, a strategy that employs stress echocardiography followed by cCTA is the second best strategy provided the prevalence of CAD is 
below 20\%. As demonstrated in figures 2 and 3, the diagnostic accuracy (FPR and FNR) of a strategy that employs stress echocardiography followed by cCTA is superior to that of stress ECG followed by cCTA.

Figure 6 demonstrates a sensitivity analysis for effective radiation dose. Figure 6A demonstrates effective radiation based upon a lower assumption of dose for stress MPS $(9.4 \mathrm{mSv})$ and cardiac catheterization $(5 \mathrm{mSv})$, while figure $6 \mathrm{~B}$ demonstrates effective radiation based upon a higher assumption of dose for cCTA (10.0mSv). Figure $6 \mathrm{C}$ demonstrates the effective radiation dose based upon a combination of the lower dose assumptions for stress MPS and cardiac catheterization with the higher assumption for cCTA. Although the overall effective radiation doses are different from those demonstrated in figure 4 , there is a similar pattern in the relative ordering of work-up strategies with respect to effective dose. Strategies that employ stress ECG or stress echocardiography alone or followed by cCTA continue to result in the lowest effective radiation dose. Similar graphs of effective radiation dose were produced when the diagnostic accuracy of cCTA was based upon the data of Miejboom (figure 6D) or Miller (figure 6E).

Figure 7 demonstrates a sensitivity analysis for expected imaging costs based upon various diagnostic accuracies of cCTA. The Miejboom values (figure 7A - lower specificity of cCTA) result in a higher FPR for cCTA, more cardiac catheterization procedures and higher overall imaging costs associated with options that include cCTA evaluation. The Miller values (figure 7B - lower sensitivity of cCTA with higher specificity) result in a lower FPR for cCTA and are associated with lower overall imaging costs. Notwithstanding the differences in absolute imaging costs, analyses based upon both the Miejboom and Miller data both 
demonstrate that imaging costs are minimized by a strategy that employs stress ECG followed by cCTA. A strategy that employs stress echocardiography followed by cCTA is the second best strategy from a cost prospective in the setting of low CAD prevalence. When the sensitivity and specificity of cCTA are set to higher values based upon the meta analysis of Vanhoenacker (figure 7C) overall imaging costs with a cCTA work-up are reduced in comparison to the baseline analysis (figure 5). 


\section{Discussion:}

We have applied a decision theoretic model to define the optimal roles of stress testing and cCTA in the work-up of the symptomatic patient with CAD. Our model demonstrates that when a stress ECG or stress echocardiogram is added prior to cCTA, the number of unnecessary cardiac catheterizations, the effective radiation dose and the imaging costs of the work-up may be minimized. The combination of stress echocardiography followed by cCTA results in a FNR that is slightly higher than stress MPS alone (up to $8 \%$ higher at a CAD prevalence of 50\%), but it minimizes the FPR of CAD diagnosis (by approximately $20 \%$ relative to MPS alone at a CAD prevalence of $10 \%$ ). Previous studies have noted that stress testing and cCTA provide complementary functional and anatomic information. ${ }^{7}$ Our study demonstrates that a combination of stress testing and cCTA may be complementary from the viewpoint of minimizing effective radiation dose and imaging costs.

The clinical application of our results is best illustrated by an example. Assume that we have a symptomatic patient with a $10 \%$ pre-test probability of clinically significant CAD (a reasonable assumption for many patients who present to the emergency department with chest pain). The least expensive options for evaluation as demonstrated in figure 5 begin with a stress ECG followed by cCTA at an expected imaging cost of $\$ 591$ or with stress echocardiography followed by cCTA at an expected cost of $\$ 807$. If the evaluation is performed only with cCTA the expected cost rises to $\$ 1412$. If the evaluation is performed only with a nuclear stress MPS test the expected cost rises to $\$ 1532$. The estimated FNR for these approaches is $3.6 \%$ for stress ECG + cCTA, $2.8 \%$ for stress echocardiography + 
cCTA, $1.2 \%$ for stress MPS alone and $0.6 \%$ for cCTA alone (figure 2). The FPR (= the rate of unnecessary cardiac catheterizations) is $3.5 \%$ for stress ECG + cCTA, $1.8 \%$ for stress echocardiography + cCTA, $21 \%$ for stress MPS alone and $15 \%$ for cCTA alone (figure 3). Expected effective radiation dose would be minimized to $1.4 \mathrm{mSv}$ by evaluation with stress echocardiography + cCTA while dose is maximized to $14.1 \mathrm{mSv}$ by evaluation with stress MPS alone (figure 4). The lower radiation dose and costs associated with stress ECG + cCTA or stress echocardiography $+\mathrm{cCTA}$ are related to the decreased FPR and greatly reduced number of unnecessary cardiac catheterizations. These cost savings and the lower morbidity related to fewer cardiac catheterizations must be weighed against a $1.6 \%$ increase in the FNR of the work-up with stress echocardiography + cCTA relative to stress MPS alone.

The most appropriate testing strategy for the work-up of symptomatic CAD depends upon the pre-test probability of disease which can be estimated on the basis of clinical presentation. ${ }^{22}$ When the pre-test probability of disease is very high the patient should be triaged directly to cardiac catheterization. For the patient described above with a low pre-test probability of disease $(\leq 20 \%)$, the combinations of stress ECG + cCTA or stress echocardiography + cCTA provide optimal options as judged by cost and radiation dose, while stress echocardiography + cCTA is optimal as judged by FPR. As the pre-test probability of CAD increases, FNR becomes a greater consideration. For a symptomatic patient with a pre-test probability of disease approaching $60 \%$, the least expensive option for evaluation is stress ECG at $\$ 1,584$, but stress ECG is associated with a FNR of $19 \%$ and is probably not appropriate. Stress ECG + cCTA has an estimated cost of $\$ 1,639$ and is associated with a FNR of $22 \%$, while stress echocardiography + cCTA has an 
estimated cost of $\$ 2,059$ and is associated with a FNR of $17 \%$. Evaluation with stress MPS alone has an estimated cost of $\$ 2,490$, a radiation dose of $16.3 \mathrm{mSv}$ and a FNR of $7.2 \%$. Evaluation with cCTA alone has an estimated cost of $\$ 2,562$, a radiation dose of $8.7 \mathrm{mSv}$ and a FNR of 3.6\%. Our analysis demonstrates that evaluation by cCTA alone is superior to all other non-invasive testing options in terms of FNR, and thus may be a more appropriate option for the patient with an intermediate pre-test probability of disease $(>20 \%)$.

In our clinical experience the MPS study is the most common form of stress test requested for evaluation of suspected CAD. Stress MPS is often advocated as superior to stress echocardiography because it is less operator dependent. Large studies suggest that the overall diagnostic performance of stress echocardiography is equivalent to that of stress MPS in the diagnosis of CAD. ${ }^{23}$ Furthermore, as demonstrated in our analysis, use of stress echocardiography reduces the false positive rate, effective radiation dose and imaging costs relative to stress MPS. Our decision analysis suggests that a combination of stress ECG with cCTA or stress echocardiography with cCTA provides an optimal work-up for symptomatic CAD in a low prevalence population, while cCTA alone may be more appropriate in an intermediate prevalence population. For the low prevalence population, if one wishes to minimize radiation dose, the stress test should be performed prior to cCTA. If the referring physician wishes to know about the anatomic presence of CAD - irrespective of whether the disease is hemodynamically significant - it may be reasonable to begin the work-up with cCTA and progress to a stress test based upon a positive cCTA.

A decision analysis model is only as good as the probability values that are 
used to define the test nodes. We have chosen generally accepted values based upon large meta analysis studies for the sensitivity and specificity of stress testing. ${ }^{10,11}$ With respect to cCTA, there is much controversy in the published literature about the true diagnostic accuracy of the technique. Furthermore, given the rapid pace of technological progress in cCTA, these numbers are constantly improving. Because the reported sensitivity and specificity with this relatively new technology varies among different centers, we chose to base our analysis on recently published multicenter trials of cCTA with cardiac catheterization as the gold standard. Unfortunately, these multicenter trials also differ in the reported values of sensitivity/specificity for cCTA. We chose to use the sensitivity/specificity values reported by Budoff for our primary calculations, ${ }^{12}$ but performed a sensitivity analysis with the values reported by Meijboom demonstrating higher sensitivity with lower specificity, ${ }^{13}$ as well as the values reported by Miller demonstrating lower sensitivity with higher specificity. ${ }^{14}$ Finally, because cCTA is a rapidly improving technology, we performed an additional analysis based upon the higher values of sensitivity/specificity reported in recent meta analyses of single center studies. ${ }^{3,4}$

When interpreting a stress test or cCTA, results are often presented on a spectrum that extends from a truly negative test to a grossly positive test. Clearly, a stress MPS study that demonstrates an ECG abnormality along with a large reversible perfusion defect is more definitive than a study that demonstrates only a small fixed perfusion deficit. Likewise a cCTA that suggests a $90 \%$ stenosis of the proximal left anterior descending artery may be more definitive than a study that suggests a 50\% lesion in the sub-optimally visualized mid-portion of the right coronary artery. In some patients, diagnostic testing with a stress test or cCTA may 
provide an indeterminate result. Nonetheless, since sensitivity/specificity values for stress testing and cCTA are available only for dichotomous test results, our decision analysis assumes a dichotomous test result (normal versus abnormal study) for stress testing, cCTA and cardiac catheterization.

Much of the cost savings of noninvasive testing results from avoiding cardiac catheterization in patients without true CAD. In addition to the benefits of reduced radiation exposure and costs, fewer cardiac catheterizations will result in reduced complications from interventional angiography. In the clinical setting, however, a mildly abnormal stress test or cCTA that does not indicate multivessel disease may not proceed to cardiac catheterization. Results of the COURAGE trial suggest that non-invasive medical therapy may be an equally appropriate option for these patients when judged in terms of mortality and future coronary events. ${ }^{24}$ Nonetheless, among the subgroup of COURAGE patients evaluated with stress MPS, patients who proceeded to cardiac catheterization and angioplasty demonstrated a reduced ischemic burden. ${ }^{25}$ Furthermore, a subsequent quality of life analysis among COURAGE participants demonstrated a small, but significant early improvement in quality of life among symptomatic patients who proceeded to cardiac catheterization and angioplasty. ${ }^{26}$ Although mildly abnormal test results may not proceed to further testing in all patients, our analysis required a standard dichotomous decision model to compute diagnostic accuracy, expected radiation exposure and costs. Based upon the results reported in the subgroup of COURAGE patients evaluated with stress MPS, we assume that a positive stress test in a symptomatic patient should trigger the performance of another test, potentially culminating in cardiac catheterization so that the patient may be treated with a stent or surgery for symptomatic relief. 
With respect to cCTA, we chose to apply a cutoff of $50 \%$ diameter stenosis as the distinction between normal and abnormal. In the cardiac catheterization laboratory, a cutoff value of $70 \%$ is more commonly used to trigger a decision for angioplasty or surgery. In many cases, the decision for intervention is based on measurement of a pressure gradient that cannot be directly predicted from the degree of anatomic stenosis. For the purpose of the current analysis, however, we are limited by the fact that most studies document the sensitivity and specificity of cCTA to predict a $50 \%$ diameter stenosis. Little data is available on the accuracy of cCTA for other levels of arterial stenosis. Of note, the Budoff study that we used to provide our baseline values for sensitivity and specificity reports the diagnostic accuracy of cCTA for both $50 \%$ and $70 \%$ diameter reduction. The sensitivity of cCTA to detect a stenosis with $\geq 50 \%$ diameter reduction is $95 \%$ while the sensitivity of cCTA to detect a stenosis with $\geq 70 \%$ diameter reduction is $94 \%$. The specificity was constant at $83 \%$ for both levels of stenosis. ${ }^{12}$ Based upon our sensitivity analysis, it is clear that this difference of $1 \%$ in sensitivity would make little difference in our overall analysis.

Although our decision tree considers the imaging costs included in the noninvasive (stress test, cCTA) and invasive (cardiac catheterization) work-up of suspected CAD, the analysis does not include future outcomes associated with correct and incorrect diagnoses of CAD. The various work-up options on the tree will result in different frequencies of cardiac catheterization and therefore in different treatment decisions for a small minority of patients. Although we present the FPR and FNR associated with each testing strategy, we do not attempt to define the cost of false positive and false negative test results. Ignoring these future costs is 
a major limitation of this study. However, although the cost of ultimate outcomes is important, this cost is very difficult to define. There is no published data to define the benefit of coronary intervention with angioplasty or surgery in patients with stenosis identified by cardiac catheterization but who have a negative stress test and/or negative cCTA. As suggested by the COURAGE trial, there is probably little mortality benefit from invasive therapy for single vessel CAD, though there may be some benefit in terms of ischemic burden and quality of life. ${ }^{25,26} \mathrm{We}$ believe that there is insufficient data to allow an accurate cost-effectiveness analysis of stress testing versus cCTA based upon long term outcomes. We have therefore decided to limit our analysis to the radiation dose and costs associated with the non-invasive and invasive diagnostic imaging components of the work-up for CAD.

In conclusion, when considering the work-up of CAD in the symptomatic patient, the FNR of cCTA is below that of stress testing at all levels of CAD prevalence. The combination of cCTA with any stress study results in a decreased FPR relative to a stress study alone. The FPR of a CAD evaluation is minimized when cCTA is employed in combination with stress echocardiography. Radiation dose is minimized by starting the work-up with stress ECG or stress echocardiography prior to cCTA. Finally, when the clinical scenario warrants further diagnostic testing for CAD, a work-up strategy that employs stress echocardiography followed by cCTA is an excellent approach to evaluate the low risk CAD patient, while cCTA alone may be more appropriate in the intermediate risk patient. 


\section{Figure Captions:}

Figure 1. Decision tree for the work-up of symptomatic CAD. The decision tree begins on the left side with a decision node - the rectangular box - where a CAD work-up strategy is chosen. The option at the very top of the tree corresponds to no advanced testing such that all patients are treated medically. The option at the bottom of the tree corresponds to cardiac catheterization for all patients. The remaining work-up options include evaluation with a stress test and/or cCTA. Each non-invasive test is identified by a round "chance" node which uses the sensitivity and specificity associated with the test to propagate positive and negative detection rates down the tree. At the terminal points of the tree (marked by arrows) the effective radiation dose and imaging cost for each pathway is computed. The focus of this study is to distinguish the strengths and weaknesses of these various work-up options.

Figure 2. False negative work-up rates for the diagnosis of CAD using baseline assumptions of sensitivity and specificity from the study of Budoff.

Figure 3. False positive work-up rates rates for the diagnosis of CAD using baseline assumptions of sensitivity and specificity from the study of Budoff.

Figure 4. Effective radiation dose for the evaluation of suspected CAD using baseline assumptions of sensitivity and specificity from the study of Budoff and effective radiation doses of $4.2 \mathrm{mSv}$ for cCTA, $12 \mathrm{mSv}$ for stress MPS and $7 \mathrm{mSv}$ for cardiac catheterization. 
Figure 5. Imaging costs for the evaluation of suspected CAD using baseline assumptions of sensitivity and specificity from the study of Budoff.

Figure 6. Sensitivity analysis for effective radiation dose in the evaluation of CAD. (A) Presents expected dose using lower estimates for effective radiation for stress MPS $(9.4 \mathrm{mSv})$ and cardiac catheterization $(5 \mathrm{mSv})$.

(B) Presents expected dose using a higher estimate for effective radiation of cCTA $(10.0 \mathrm{mSv})$.

(C) Presents expected dose using lower estimates for effective radiation for stress MPS $(9.4 \mathrm{mSv})$ and cardiac catheterization $(5 \mathrm{mSv})$ in combination with a higher estimate for effective radiation of cCTA $(10.0 \mathrm{mSv})$.

(D) Presents expected dose based upon the data of Miejboom and our baseline assumptions of effective radiation doses of $4.2 \mathrm{mSv}$ for cCTA, $12 \mathrm{mSv}$ for stress MPS and $7 \mathrm{mSv}$ for cardiac catheterization.

(E) Presents expected dose based upon the data of Miller and our baseline assumptions of effective radiation doses of $4.2 \mathrm{mSv}$ for cCTA, $12 \mathrm{mSv}$ for stress MPS and $7 \mathrm{mSv}$ for cardiac catheterization.

Figure 7. Sensitivity analysis for expected imaging costs in the evaluation of CAD. (A) Presents expected imaging costs based upon the sensitivity and specificity data of Miejboom.

(B) Presents expected imaging costs based upon the sensitivity and specificity data of Miller.

(C) Presents expected imaging costs based upon higher values of sensitivity and specificity in the meta analysis of Vanhoenacker. 


\section{References}

\footnotetext{
${ }^{1}$ Heron M, Hoyert DL, Murphy SL, Xu J, Kochanek KD, Tejada-Vera B. National Vital Statistics Reports - Deaths: final data for 2006. Vol 57, No. 14. April 2009. Centers for Disease Control and Prevention. http://www.cdc.gov/nchs/data/nvsr/nvsr57/nvsr57_14.pdf

${ }^{2}$ Lee TH, Boucher CA. Clinical practice. Noninvasive tests in patients with stable coronary artery disease. N Engl J Med. 2001 Jun 14;344(24):1840-5.

${ }^{3}$ Vanhoenacker PK, Heijenbrok-Kal MH, Van Heste R, Decramer I, Van Hoe LR, Wijns W, Hunink MG. Diagnostic performance of multidetector CT angiography for assessment of coronary artery disease: meta-analysis. Radiology 2007; 244(2):419-28.

${ }^{4}$ Meijer AB, O YL, Geleijns J, Kroft LJ. Meta-analysis of 40- and 64-MDCT angiography for assessing coronary artery stenosis. AJR Am J Roentgenol. 2008; 191(6):1667-75.

${ }^{5}$ Hacker M, Jakobs T, Hack N, Nikolaou K, Becker C, von Ziegler F, Knez A, König A, Klauss V, Reiser M, Hahn K, Tiling R. Sixty-four slice spiral CT angiography does not predict the functional relevance of coronary artery stenoses in patients with stable angina. Eur J Nucl Med Mol Imaging. 2007 Jan;34(1):4-10.

${ }^{6}$ Gaemperli O, Schepis T, Valenta I, Koepfli P, Husmann L, Scheffel H, Leschka S, Eberli FR, Luscher TF, Alkadhi H, Kaufmann PA. Functionally relevant coronary artery disease: comparison of 64-section CT angiography with myocardial perfusion SPECT. Radiology. 2008;248(2):414-23

${ }^{7}$ Schuijf JD, Bax JJ. CT angiography: an alternative to nuclear perfusion imaging? Heart 2008; 94(3):255-7.

${ }^{8}$ Budoff MJ, Rasouli ML, Shavelle DM, Gopal A, Gul KM, Mao SS, Liu SH, McKay CR. Cardiac CT angiography (CTA) and nuclear myocardial perfusion imaging (MPI)-a comparison in detecting significant coronary artery disease.
} Acad Radiol. 2007; 14(3):252-7.

${ }^{9}$ Hendel RC, Patel MR, Kramer CM, Poon M, Hendel RC, Carr JC, Gerstad NA, Gillam LD, Hodgson JM, Kim RJ, Kramer CM, Lesser JR, Martin ET, Messer JV, Redberg RF, Rubin GD, Rumsfeld JS, Taylor AJ, Weigold WG, Woodard PK, Brindis RG, Hendel RC, Douglas PS, Peterson ED, Wolk MJ, Allen JM, Patel MR; American College of Cardiology Foundation Quality Strategic Directions Committee Appropriateness Criteria Working Group; American College of Radiology; Society of Cardiovascular Computed Tomography; Society for Cardiovascular Magnetic Resonance; American Society of Nuclear Cardiology; North American Society for Cardiac Imaging; Society for Cardiovascular Angiography and Interventions; Society of Interventional Radiology.

ACCF/ACR/SCCT/SCMR/ASNC/NASCI/SCAI/SIR 2006 appropriateness criteria for cardiac computed tomography and cardiac magnetic resonance imaging: a report of the American College of Cardiology Foundation Quality Strategic Directions Committee Appropriateness Criteria Working Group, American College of Radiology, Society of Cardiovascular Computed Tomography, Society for Cardiovascular Magnetic Resonance, American Society of Nuclear Cardiology, North American Society for Cardiac Imaging, Society for Cardiovascular Angiography and Interventions, and Society of Interventional Radiology. J Am Coll Cardiol. 2006 Oct 3;48(7):1475-97.

${ }^{10}$ Gianrossi R, Detrano R, Mulvihill D, Lehmann K, Dubach P, Colombo A, McArthur D, Froelicher V. Exerciseinduced ST depression in the diagnosis of coronary artery disease. A meta-analysis. Circulation 1989 Jul;80(1):87-98.

${ }^{11}$ Garger AM, Solomon NA. Cost-effectiveness of alternative test strategies for the diagnosis of coronary artery disease. Ann Intern Med. 1999; 130(9):719-28.

${ }^{12}$ Budoff MJ, Dowe D, Jollis JG, Gitter M, Sutherland J, Halamert E, Scherer M, Bellinger R, Martin A, Benton R, Delago A, Min JK. Diagnostic performance of 64-multidetector row coronary computed tomographic angiography for evaluation of coronary artery stenosis in individuals without known coronary artery disease: results from the prospective multicenter ACCURACY (Assessment by Coronary Computed Tomographic Angiography of Individuals Undergoing Invasive Coronary Angiography) trial. J Am Coll Cardiol 2008 Nov 18;52(21):1724-32.

${ }^{13}$ Meijboom WB, Meijs MF, Schuijf JD, Cramer MJ, Mollet NR, van Mieghem CA, Nieman K, van Werkhoven JM, Pundziute G, Weustink AC, de Vos AM, Pugliese F, Rensing B, Jukema JW, Bax JJ, Prokop M, Doevendans PA, Hunink MG, Krestin GP, de Feyter PJ. Diagnostic accuracy of 64-slice computed tomography coronary angiography. J Am Coll Cardiol 2008 Dec 16;52(25):2135-44. 
${ }^{14}$ Miller JM, Rochitte CE, Dewey M, Arbab-Zadeh A, Niinuma H, Gottlieb I, Paul N, Clouse ME, Shapiro EP, Hoe J, Lardo AC, Bush DE, de Roos A, Cox C, Brinker J, Lima JA. Diagnostic performance of coronary angiography by 64row CT. N Engl J Med 2008 Nov 27;359(22):2324-36.

${ }^{15}$ Valentin J, Valentin DJ. Radiation dose to patients from radiopharmaceuticals: (addendum 2 to ICRP publication 53) approved by the commission in September 1997. Ann ICRP 1998;28:1-123.

${ }^{16}$ Mettler FA Jr, Huda W, Yoshizumi TT, Mahesh M. Effective doses in radiology and diagnostic nuclear medicine: a catalog. Radiology 2008 Jul;248(1):254-63.

${ }^{17}$ Hirai N, Horiguchi J, Fujioka C, Kiguchi M, Yamamoto H, Matsuura N, Kitagawa T, Teragawa H, Kohno N, Ito K. Prospective versus retrospective ECG-gated 64-detector coronary CT angiography: assessment of image quality, stenosis, and radiation dose. Radiology 2008 Aug;248(2):424-30.

${ }^{18}$ Shuman WP, Branch KR, May JM, Mitsumori LM, Lockhart DW, Dubinsky TJ, Warren BH, Caldwell JH. Prospective versus retrospective ECG gating for 64-detector CT of the coronary arteries: comparison of image quality and patient radiation dose. Radiology 2008 Aug;248(2):431-7.

${ }^{19}$ Raff GL, Chinnaiyan KM, Share DA, Goraya TY, Kazerooni EA, Moscucci M, Gentry RE, Abidov A; Advanced Cardiovascular Imaging Consortium Co-Investigators. Radiation dose from cardiac computed tomography before and after implementation of radiation dose-reduction techniques. JAMA. 2009 Jun 10;301(22):2340-8.

${ }^{20}$ Coles DR, Smail MA, Negus IS, Wilde P, Oberhoff M, Karsch KR, Baumbach A. Comparison of radiation doses from multislice computed tomography coronary angiography and conventional diagnostic angiography. J Am Coll Cardiol. 2006 May 2;47(9):1840-5.

${ }^{21}$ Gerber TC, Carr JJ, Arai AE, Dixon RL, Ferrari VA, Gomes AS, Heller GV, McCollough CH, McNitt-Gray MF, Mettler FA, Mieres JH, Morin RL, Yester MV. Ionizing radiation in cardiac imaging: a science advisory from the American Heart Association Committee on Cardiac Imaging of the Council on Clinical Cardiology and Committee on Cardiovascular Imaging and Intervention of the Council on Cardiovascular Radiology and Intervention. Circulation. 2009 Feb 24;119(7):1056-65.

22 Diamond GA, Forrester JS. Analysis of probability as an aid in the clinical diagnosis of coronary-artery disease. N Engl J Med 1979;300(24):1350-8.

${ }^{23}$ Heijenbrok-Kal MH, Fleischmann KE, Hunink MG. Stress echocardiography, stress single-photon-emission computed tomography and electron beam computed tomography for the assessment of coronary artery disease: a metaanalysis of diagnostic performance. Am Heart J. 2007 Sep;154(3):415-23.

24 Boden WE, O'Rourke RA, Teo KK, Hartigan PM, Maron DJ, Kostuk WJ, Knudtson M, Dada M, Casperson P, Harris CL, Chaitman BR, Shaw L, Gosselin G, Nawaz S, Title LM, Gau G, Blaustein AS, Booth DC, Bates ER, Spertus JA, Berman DS, Mancini GB, Weintraub WS; COURAGE Trial Research Group. Optimal medical therapy with or without PCI for stable coronary disease. N Engl J Med. N Engl J Med. 2007; 356(15):1503-16.

${ }^{25}$ Shaw LJ,Berman DS,Maron DJ, Mancini GB, Hayes SW, Hartigan PM, Weintraub WS, O'Rourke RA, Dada M, Spertus JA, Chaitman BR, Friedman J, Slomka P, Heller GV, Germano G, Gosselin G, Berger P, Kostuk WJ, Schwartz RG, Knudtson M, Veledar E, Bates ER, McCallister B, Teo KK, Boden WE; COURAGE Investigators. Optimal medical therapy with or without percutaneous coronary intervention to reduce ischemic burden: Results from the clinical outcomes utilizing revascularization and aggressive drug evaluation (COURAGE) trial nuclear substudy. Circulation 2008; 117: 1283-1291.

${ }^{26}$ Weintraub WS, Spertus JA, Kolm P, Maron DJ, Zhang Z, Jurkovitz C, Zhang W, Hartigan PM, Lewis C, Veledar E, Bowen J, Dunbar SB, Deaton C, Kaufman S, O'Rourke RA, Goeree R, Barnett PG, Teo KK, Boden WE; COURAGE Trial Research Group, Mancini GB. Effect of PCI on quality of life in patients with stable coronary disease. N Engl J Med. 2008; 359(7):677-87. 